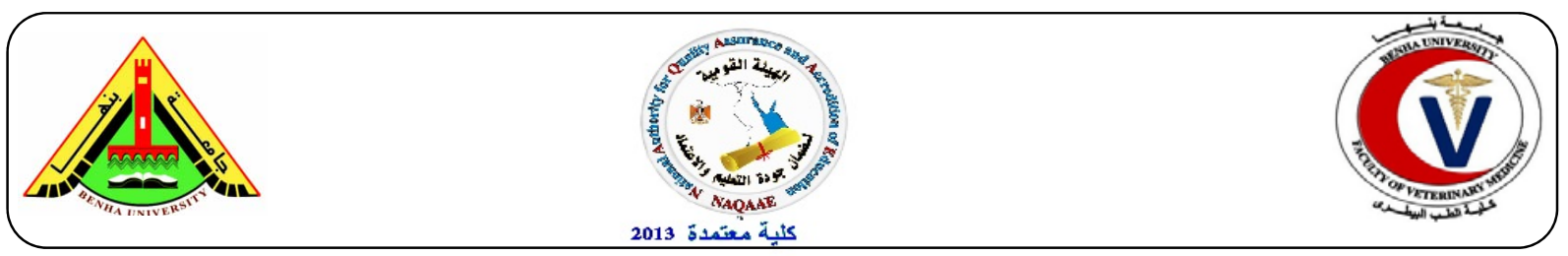

\title{
Biochemical studies on the effect of curcumin in experimentally induced osteoarthritis in rats
}

\author{
Yakuot A. El-senosi ${ }^{1}$, Ashraf A. El komy ${ }^{2}$ and MoKhtar, $A^{1}$. \\ 1-Biochemistry department, Faculty of Vet. Med. Moshtohor, Benha University. \\ 2- Pharmacology department, Faculty of Vet. Med. Moshtohor, Benha University.
}

\begin{abstract}
A B S T R A C T
Osteoarthritis (OA) is the most common disease of joints in adults around the world. Osteoarthritis was induced in rats by intra-articular injection of $2 \mathrm{mg} / \mathrm{kg}$ b.wt of monosodium-iodoacetate (MIA). Thirty six male, albino rats were divided into four equal groups. $1^{\text {st }}$ group was not subjected to any intervention and was used as normal control. $2^{\text {nd }}$ group (OA induced group) received injection of MIA intra-articular. $3^{\text {rd }}$ group ( OA + curcumin treated group) injected MIA and treated with $200 \mathrm{mg} / \mathrm{kg}$ b.wt of curcumin orally and daily / 28 day. $4^{\text {th }}$ group ( curcumin normal group) received 200 $\mathrm{mg} / \mathrm{kg}$ b.wt of curcumin orally and daily / 28 day. Blood samples were collected at $28^{\text {th }}$ day. The obtained results showed a significant increase in MDA level and SOD activity in MIA induced OA. On the other hand, a significant decrease in GSH, GPx, CAT and GAG were observed in OA induced rats. Curcumin treatment in OA induced rats significantly increased GSH concentration, GPx, CAT and GAG activities with marked decrease in MDA concentration. In conclusion, the present study demonstrated that curcumin administration provided an effective protection and treatment against OA and oxidative damage in MIA injected rats.
\end{abstract}

Key Words: OA, curcumin, inflammatory mediators, oxidative damage.

(http://www.bvmj.bu.edu.eg)

(BVMJ-33(2): 46-51, DECEMBER, 2017)

\section{INTRODUCTION}

Osteoarthritis is a common degenerative disorder of the articular cartilage associated with hypertrophic changes in the bone (Goodman, 2005). OA has a multifactorial etiology and can be considered the product of an inter play between systemic and local factors. For example, a person may have an inherited predisposition to develop OA but may only develop it if an insult to the joint has occurred. The relative importance of risk factors may vary for different joints, for different stages of the disease, for the development as opposed to the progression of disease, and for radiographic versus symptomatic disease. There is even some evidence suggesting that risk factors may act differently according to individual radiographic features, such as osteophytes and joint space narrowing (Felson et al., 2000). Intraarticular injection of MIA was chosen because it has been shown to produce long-lasting OA of the knee joint in rodents (Bove et al., 2003). In addition to inhibiting lipid peroxidation, curcumin demonstrates free radical-scavenging activity. It has been shown to scavenge various reactive oxygen species produced by macrophages (including superoxide anions, hydrogen peroxide and nitrite radicals) both in vitro as well as in vivo using rat peritoneal macrophages as a model (Joe et al.,1994). Interestingly, curcumin not only exhibits antioxidative and free radical scavenging properties, but also enhances the activity of other antioxidants, such as superoxide dismutase, catalase, and glutathione peroxidase (Reddy and Lokesh, 1994).

Accordingly, the aim of the present study was designed to investigate the potential beneficial effect of curcumin on an experimental rat model of MIA-induced OA through evaluation of some biochemical blood parameters: super oxide dismutase (SOD), catalase (CAT), sulfated glycosaminoglycan (s-GAG), glutathione peroxidase (GPx), reduced glutathione $(\mathrm{GSH})$ and malondialdehyde (MDA).

\section{MATERIAL AND METHODS}

\subsection{Experimental Animals:}

Thirty-six, 12-weeks old male, albino rats and average body weight 100_150 g were used in this 
study. Rats were obtained from laboratory Animal research Centre, Faculty of Veterinary Medicine, Benha University. The animals were fed on constant ration, water and ad libitum were supplied.

\subsection{Experimental design:}

Rats were divided into four equal groups, each group contained 9 rats as follow: Group 1: (control normal group) were not subjected to any intervention and were used as controls. Group 2: (OA - induced group) received intra-articular injection of $2 \mathrm{mg} / \mathrm{kg}$ b.wt of monoiodoacetate (MIA) (Crystal Powder M=185.96g/mol, Germany, Sigma) and OA was induced in the right knees joint of rats according to the method of ( Bove et al., 2003). Group 3: (OA+ curcumin treated group) injected of $2 \mathrm{mg} / \mathrm{kg}$ b.wt MIA intra-articular and were treated with $200 \mathrm{mg} / \mathrm{kg}$ b.wt of curcumin orally and daily for 28 day. Group 4: (curcumin normal group) creceived 200 $\mathrm{mg} / \mathrm{kg}$ b.wt of curcumin orally and daily for 28 day.

\subsection{Sampling:}

Blood samples and articular tissue specimen were collected once, 24 hours after the end of $28^{\text {th }}$ day, from all animal groups (control and experimental groups).

\subsection{Blood Samples:}

At the end of the experiment, blood samples were collected after overnight fasting from the retro-orbital venous plexus located at the medial canthus of the eye using heparinized capillary tubes, in dry, clean screw-copped tubes then allowed to coagulate at room temperature for 30 minutes and separated by centrifugation at 3000 r.p.m. for 15 minutes. The clean, clear serum was separated by automatic micropipettes and received in dry sterile Eppendorf's tubes.

\subsection{Biochemical analysis:}

L-malondialdehde (MDA), Reduced Glutathione (GSH), super oxide dismutase (SOD), glutathione peroxidase(GPx), sulfated glycosaminoglycan (s-GAG) and catalase (CAT) were determined according to the method described by (Mitsuru and Midori, 1978), (Ellman, 1959), (Nandi and Chatterjee, 1988), (Lawrence and burk, 1976), (Biocolor Ltd., 2007) and (Aebi,1974) respectively.

\subsection{Statistical Analysis}

The obtained data were analyzed represented using the statistical package for social science (SPSS, 13.0 software, 2009), for obtaining mean and standard deviation and error. The data were analyzed using one-way ANOVA to determine the statistical significance of differences among groups. Duncan's test was used for making a multiple comparison among the groups for testing the inter-grouping.

\section{RESULTS}

The obtained results in table (1) showed a significant decrease in GSH concentration was observed in osteoarthritic induced group when compared to the normal control one. Treatment with curcumin in osteoarthritic group cause a significant increase in GSH level as compared to osteoarthritic one. There was no significant increase in GSH was observed in curcumin normal treated rats compared with normal control group.

Moreover, there was a significant increase in MDA level was observed in osteoarthritic induced group when compared to the normal control one. Treatment with curcumin in osteoarthritic group cause a significant decrease in MDA level as compared to osteoarthritic one. There was no significant increase in MDA was observed in curcumin normal treated rats compared with normal control group.

In addition, there was a significant decrease in GPx activity was observed in osteoarthritic induced group when compared to the normal control one. Treatment with curcumin in osteoarthritic group cause a significant increase in GPx activity as compared to osteoarthritic one. There was no significant increase in GPx was observed in curcumin normal treated rats compared with normal control group.

Also, there was a significant increase in SOD activity activity was observed in osteoarthritic induced group when compared to the normal control one. Treatment with curcumin in osteoarthritic group cause no significant decrease in SOD activity as compared to osteoarthritic one. There was no significant decrease in SOD was observed in curcumin normal treated rats compared with normal control group.

Moreover, there was a significant decrease in CAT activity was observed in osteoarthritic induced group when compared to the normal control one. Treatment with curcumin in osteoarthritic group cause a significant increase in CAT activity as compared to osteoarthritic one. There was a significant increase in CAT was observed in curcumin normal treated rats compared with normal control group. 
Finally, there was a significant decrease in GAG activity was observed in osteoarthritic induced group when compared to the normal control one. Treatment with curcumin in osteoarthritic group cause a significant increase in
GAG activity as compared to osteoarthritic one. There was no significant increase in GAG was observed in curcumin normal treated rats compared with normal control group.

Table (1): Effect of curcumin treated on serum sulfated Glycosaminoglycan, oxidants and antioxidants osteoarthritis induced rats.

\begin{tabular}{lcccc}
\hline \multicolumn{1}{c}{ Parameters } & G1 & G2 & G3 & G4 \\
\hline $\begin{array}{l}\text { GSH } \\
(\mathrm{mM} / \mathrm{g} \text { tissue })\end{array}$ & $48.6 \pm 6.5^{\mathrm{a}}$ & $22.1 \pm 3.6^{\mathrm{b}}$ & $35.2 \pm 5.6^{\mathrm{c}}$ & $52.3 \pm 7.3^{\mathrm{a}}$ \\
$\begin{array}{l}\text { MDA } \\
(\mathrm{mM} / \mathrm{g} \text { tissue })\end{array}$ & $9.3 \pm 2.1^{\mathrm{a}}$ & $25.2 \pm 3.2^{\mathrm{b}}$ & $16.5 \pm 3.4^{\mathrm{c}}$ & $9.5 \pm 2.6^{\mathrm{a}}$ \\
$\begin{array}{l}\text { GPx } \\
(\mathrm{U} / \mathrm{mg} \text { protein })\end{array}$ & $10.5 \pm 1.3^{\mathrm{a}}$ & $3.9 \pm 0.6^{\mathrm{b}}$ & $6.8 \pm 1.1^{\mathrm{c}}$ & $13.1 \pm 2.5^{\mathrm{a}}$ \\
& & & & \\
SOD & $3.12 \pm 0.5^{\mathrm{a}}$ & $7.17 \pm 1.3^{\mathrm{b}}$ & $6.1 \pm 0.9^{\mathrm{b}}$ & $2.5 \pm 0.6^{\mathrm{a}}$ \\
$(\mathrm{U} / \mathrm{mg}$ protein $)$ & & & & \\
$\begin{array}{l}\text { CAT } \\
(\mathrm{U} / \mathrm{mg} \text { protein })\end{array}$ & $12.75 \pm 0.9^{\mathrm{a}}$ & $6.19 \pm 0.7^{\mathrm{b}}$ & $11.2 \pm 0.4^{\mathrm{a}}$ & $16.1 \pm 0.5^{\mathrm{d}}$ \\
$\begin{array}{l}\mathrm{GAG} \\
(\mathrm{mM} / \mathrm{g} \text { tissue })\end{array}$ & $42.2 \pm 9.5^{\mathrm{a}}$ & $39.6 \pm 8.9^{\mathrm{b}}$ & $43.5 \pm 11.1^{\mathrm{a}}$ & $42.6 \pm 13.2^{\mathrm{a}}$ \\
\hline
\end{tabular}

Data are presented as (Mean \pm S.E). S.E $=$ Standard error. Mean values with different superscript letters in the same column are significantly different at $(P \leq 0.05)$. (G1): Normal control group. (G2): OA- induced group. (G3): OA+ curcumin treated group. (G4): Curcumin normal group.

\section{DISCUSSION}

The obtained results demonstrated in table (1) showed a significant decrease in GSH level in osteoarthritic rats when compared with normal control group. These results were nearly similar to these reported by Regan et al., (2008) who reported that, was a non-significant decrease in GSH level. A significant increase in GSH concentration was noticed in curcumin treated osteoarthritic group when compared with osteoarthritic one. Similarly, to Ramadan et al., (2010) showed that, a significant increase in GSH level was observed in arthritic rats received curcumin especially from the day of arthritis induction that emphasizes the role of curcumin in preventing organs damage and bone loss in adjuvant induced arthritic rat model through scavenging the free radicals. Also, Biswas et al.,(2005) reported that, there was an increase in GSH level in curcumin-treated cells as compared with untreated cells. The value of L-MDA concentration was significantly increased in in osteoarthritic rats when compared with normal control group. The obtained results are nearly similar with Rubyk et al.,(1988) who reported that a significant increase in serum MDA level was observed in OA. Thus, these findings were in keeping with possible evidence of free radical production and damage in OA.

However, a significant decrease in MDA concentration was observed in curcumin treated osteoarthritic group. These results were nearly similar to Maria et al., (2013) who illustrated that, a significant efficiency of curcumin was present in the initial phase of advancing inflammation, and the late persistency of elevated MDA concentration suggested a breakdown of the defensive potential. Also, Zheng et al., (2009) reported that, curcumin caused a significant decrease the value of MDA concentration. The obtained results revealed that, a significant decrease in GPx activity was observed in osteoarthritic rats when compared with normal control group. The obtained results agree well with those reported by Ostalowska et al., (2006) and Erturk et al.,(2012) who recorded that, the activity of antioxidant enzymes such as SOD, CAT and GPX were decreased in OA patients, confirming the role of oxidative stress in OA pathogenesis. Chondrocytes expressed a variety of antioxidant defenses against the harmful effects of ROS, including CAT, SOD and GPx. On the other hand a significant increase in GPx activity was observed 
in curcumin treated osteoarthritic group when compared with osteoarthritic one. Similarly, Cemal et al.,(2016) showed that, curcumin treatment significantly increased GPx activity compared to the saline-treated group. On Contrary, Kalpravidh et al.,(2010) demonstrated that, curcuminoids treatment for 12 months significantly decreased the activity of GPx in RBC. The RBC GPx activity was gradually reduced during 12 months of curcuminoids treatment and returned to baseline after the withdrawal of curcuminoids in $\beta$ thalassemia/Hb E patients.

Regarding SOD activity, the obtained results showed a significant increase in SOD activity in osteoarthritic rats when compared with normal control group. A non significant decrease in SOD activity was observed in osteoarthritic curcumin treated group when compared with osteoarthritic one. Contariwise, Cemal et al.,(2016) said that, the curcumin-treated rats exhibited significantly higher SOD activity than the methylprednisolonetreated animals. El-Agamy,(2010) demonstrated that, there was a significant increase of SOD activity in the curcumin-treated group when compared to aflatoxin B1- intoxicated group. The obtained results revealed that, there was a significant decrease in CAT activity in osteoarthritic rats when compared with normal control group. Also, Hassan et al.,(2001) reported that, the uncontrolled production of reactive oxygen species ( ROS) (especially superoxide anion and hydroxyl radicals) by phagocytic cells due to the inflammatory surge in rheumatoid arthritis (RA) leads to a decrease in SOD and CAT activities in addition to GSH level as a consequence of their super saturation and consumption during oxidative stress as well as loss through cellular lysis, which resulted from membrane lipid peroxidation and lysosomal destruction. There was a significant increase in CAT activity in osteoarthritic treated group with curcumin when compared with osteoarthritic one. Contrary to this result, Farghaly and Hussein, (2010) reported that, the activities of CAT, GST, NADPH-dependent GR and NADH-dependent GR in liver were significantly lower in curcumin treated rats against paracetamol-induced liver damage.

A significant decrease in GAG concentration was observed in osteoarthritic rats when compared with normal control group. On contrary, Alwan., (1991) reported that, Synovial fluids from joints with OA showed a very highly level significant of GAG. Serum and urine also showed increased levels of GAG which were significantly different from normal but the amounts were very much less than those found acutely infected joints and normal joints. The levels of GAG in septic arthritis were lower than those in OA. This may be due to the collection of samples at an early stage of the disease before considerable damage had occurred. Serum and urine concentrations of GAG were low compared to synovial fluid. This was probably because these molecules were rapidly taken up and degraded by liver endothelial cells (Smedsrod, 1985). There was a significant increase in GAG activity observed in osteoarthritic treated group with curcumin when compared with osteoarthritic one. In like manner, Hosseininia et al., (2013) revealed that, the amount of GAG per dry weight was higher in OA samples compared to reference samples, which suggests a capacity for GAG biosynthesis in hip OA cartilage.

\section{CONCLUSION AND RECOMMENDATION}

In conclusion, the present study demonstrated that curcumin administration provided an effective protection and treatment against $\mathrm{OA}$ and oxidative damage in MIA injected rats. Since, curcumin was able to ameliorate serum biochemical parameters, enzymatic and non-enzymatic antioxidant defense system in blood. The results indicate that curcumin exerts protective effect by restoring the activities of joint biomarker enzymes and reversing the oxidant-antioxidant imbalance during MIAinduced OA. We recommended that, administration of diet rich in curcumin is very important for treatment and protection of different articular tissue, especially joint tissue, against oxidative stress or even inflammation.

\section{REFERENCES}

Aebi,H.(1980): Enzymes1: oxidoreductases, transferases. In: Bergmeyer $\mathrm{H}, \mathrm{Ed}$. Methods of enzymatic analysis, Deerfield Beach, FL: Verlag; vol. III; p: 273-282.

Alwan, W.; Carter, S.; Bennett,D and Edwards, G.( 1991): Glycosaminog lycans in horses with osteoarthritis. Equine ve!. J. 23 (I) 4447.

Biocolor Ltd. (2007): Blyscan ${ }^{\mathrm{TM}}$ Sulfated Glycosaminoglycan Assay.8 Meadowbank Road, Carrickfergus BT38 8YFNorthern Ireland.1-20.

Biswas,S.; McClure, D.; Jimenez, L.; Megson, I and Rahman, I.(2005): Antioxid Redox Signal. Curcumin induces glutathione biosynthesis and inhibits NF-kappa B activation and interleukin- 8 release in 
alveolar epithelial cells: mechanism of free radical scavenging activity. (1-2):32-41.

Bove, S.; Calcaterra, S.; Brooker, R.; Huber, C.; Guzman, R.; Juneau, P.; Schrier, D and Kilgore, K. (2003): Weight bearing as a measure of disease progression and efficacy of anti-inflammatory compounds in a model of monosodium iodoacetate-induced osteoarthritis. Osteoarth Cart. 11:821-830.

Cemal,E.; Kahveci,R.; Gokce, A.; Fevzi,M.; Kisa, U.; Aksoy, N.; Cemil, B and Erdogan, B. (2016): Curcumin Attenuates Inflammation, Oxidative Stress, and Ultrastructural Damage Induced by Spinal Cord IschemiaReperfusion Injury in Rats. Journal of Stroke and Cerebrovascular Diseases, Vol. 25, No. 5;pp 1196-1207.

Ellman,G. (1959) : Tissue sulfhydryl groups, ARCH. BIOCHEM. BIOPHYS, vol. 82, $70-$ 77.

El-Agamy,D.(2010): Comparative eVects of curcumin and resveratrol on aflatoxin B1induced liver injury in rats. Arch Toxicol ,84:389-396

Erturk,C.; Altay,M.; Selek,S and Kocyigit,A. (2012):

Paraoxonase- 1 activity and oxidative status in patients with knee osteoarthritis and their relationship with radiological and clinical parameters, Scand. J. Clin. Lab. Invest. 72 ;433-439.

Farghaly,H and Hussein,M. (2010): Protective Effect of Curcumin Against Paracetamolinduced Liver Damage. Australian Journal of Basic and Applied Sciences, 4(9): 42664274.

Felson, D.; Couropmitree, N.; Chaisson, C.; Hannan, M.; Zhang, Y.; Mcalindon, T.; Lavallet, M.; Levy, D and Myers, R. (1998): Evidence for a Mendelian gene in a segregation analysis of generalized radiographic osteoarthritis: The official Journal of The American College of Rheumatology. Arthritis Rheum. 41(6):1064-71.

Goodman,S. (2005): Osteoarthritis. In: Yee A, Paget S, eds. Expert Guide to Rheumatology. Philadelphia, Pa.: American College of Physicians:269-283.

Hassan,M.; Hadi,R.; Al-Rawi,Z.; Padron,V and Stohs,S.(2001): The glutathione defense system in the pathogenesis of rheumatoid arthritis. Journal of Applied Toxicology 21(1): 69-73.

Hosseininia,S.; Lindberg,L and Dahlberg,L.(2013): Cartilage collagen damage in hip osteoarthritis similar to that seen in knee osteoarthritis; a case-control study of relationship between collagen, glycosaminoglycan and cartilage swelling. http://www.biomedcentral.com/14712474/14/18.

Joe, B and Lokesh, B. (1994): Role of capsaicin, curcumin and dietary $<\mathrm{i}>\mathrm{n}-3$ fatty acids in lowering the generation of reactive oxygen species in rat peritoneal macrophages. Biochimica et Biophysica Acta (BBA)Molecular Cell Research. 1224(2):255-263. 51.

Kalpravidh, R.; Siritanaratkul, N.; Insain, P.; Charoensakdi, R.; Panichkul, N.; Hatairaktham, S.; Srichairatanakool, S.; Phisalaphong, C.; Rachmilewitz, E and Fucharoen, S. (2010): Improvement in oxidative stress and antioxidant parameters in $\beta$-thalassemia/Hb E patients treated with curcuminoids. Clinical Biochemistry 43, 424-429.

Lawrence,R and Burk, R.(1976): Glutathione peroxidase activity in selenium-deficient rat liver. 425(3):5039.

Maria, B.; Marilena, O.; Cezar, C and Nicoleta, D. ( 2013):The effects of silicon dioxide and curcumin on malondialdehyde found in lung tissue $A M T$, v. II, no. 3, 2013, p. 296.

Mitsuru, U and Midori, M.(1978): Determination of Malonaldhyde precursor in tissues by Thiobarbituric Acid Test. National Institute of Hygienic Sciences, l-18-1, Komiyoga, Setagttya, Tokyo 158, Japan . An analytical biochemistry $86,271-278$.

Nandi,A and Chatterjee,I.( 1988):Assay of superoxide dismutase activity in animal tissues. Journal of Biosciences. 13,310_315.

Ostalowska, A.; Birkner, E.; Wiecha, M.; Kasperczyk, S.; Kasperczyk, A.; Kapolka, D and Zon-Giebel, A.(2006): Lipid peroxidation and antioxidant enzymes in synovial fluid of patients with primary and secondary osteoarthritis of the knee joint, Osteoarthr. Cartil. 14;139-145.

Ramadan, G; Ali,M and Mohamed, W. (2010): Anti-inflammatory and Anti-oxidant Properties of Curcuma longa (Turmeric) Versus Zingiber officinale (Ginger) Rhizomes Adjuvant-Induced Arthritis in Rat. DOI. 10.1007/s10753-010-9278-0.

Reddy, A and Lokesh,B. (1994): Effect of dietary turmeric (Curcuma longa) on iron-induced lipid peroxidation in the rat liver. Food Chem. Toxicol., 32:279-283.

Regan, E.; Bowler,R and Crapo,J. (2008): Joint fluid antioxidants are decreased in 
osteoarthritic joints compared to joints with macroscopically intact cartilage and subacute injury. Osteoarthr. Cartil. 16, $515-21$.

Rubyk, B.; Fil'chagin, $M$ and Sabadyshin, R. (1988): Change in lipid peroxidation in patients with primary osteoarthrosis deformans. Ter Arkh, 60 (9): 110-113.

Smedsrod, B., Kjellen, L and Pertoft, H. (1985): Endocytosis and degradation of chondroitin sulphate by liver endothelial cells. Biochem. J. 229, 63-71.

Stastical Analysis System (S.A.S); (2009): version 9-00. S.A. S Institute Inc., cary,NC,USA.

Zheng, Y.; Yu,L.; Zhang,Y.; Ye, Gand Yi, J. (2009): Effects of curcumin on malondialdehyde and c-fos protein in hypoxia ischemia brain tissue in rats. $\mathrm{Fa} \mathrm{Yi}$ Xue Za Zhi. 25(1):6-8. 